\title{
Abnormal Blood Oxygen Level-Dependent Fluctuations in Focal Cortical Dysplasia and the Perilesional Zone: Initial Findings
}

\author{
(D) L. Gupta, (DP.A.M. Hofman, DR.M.H. Besseling, (D).F.A. Jansen, and (D)W.H. Backes
}

\begin{abstract}
BACKGROUND AND PURPOSE: Focal cortical dysplasia is a common cause of intractable epilepsy for which neurosurgery is an option. Delineations of a focal cortical dysplasia lesion on structural brain images may not necessarily reflect the functional borders of normal tissue. Our objective was to determine whether abnormalities in spontaneous blood oxygen level-dependent fluctuations arise in focal cortical dysplasia lesions and proximal regions.
\end{abstract}

MATERIALS AND METHODS: Fourteen patients with focal cortical dysplasia-related epilepsy and 16 healthy controls underwent structural and resting-state functional MR imaging. Three known blood oxygen level-dependent measures were determined, including the fractional amplitude of low-frequency fluctuations, regional homogeneity, and wavelet entropy. These measures were evaluated in the lesion and perilesional zone and normalized to the contralateral cortex of patients with focal cortical dysplasia and healthy controls.

RESULTS: Patients showed significantly decreased fractional amplitude of low-frequency fluctuations and increased wavelet entropy in the focal cortical dysplasia lesion and the perilesional zone $(\leq 2 \mathrm{~cm})$ relative to the contralateral homotopic cortex and the same regions in healthy controls. Regional homogeneity was significantly increased in the focal cortical dysplasia lesion compared with the contralateral homotopic cortex and healthy controls.

CONCLUSIONS: Abnormalities in spontaneous blood oxygen level-dependent fluctuations were seen up to $2 \mathrm{~cm}$ distant from the radiologically visible boundary. It was demonstrated that functional boundaries go beyond structural boundaries of focal cortical dysplasia lesions. Validation is required to reveal whether this information is valuable for surgical planning and outcome evaluation of focal cortical dysplasia lesions and comparing current results with electrophysiologic analysis.

ABBREVIATIONS: $B$ OLD = blood oxygen level-dependent; $E E G=$ electroencephalography; fALFF $=$ fractional amplitude of low-frequency fluctuations; $F C D=$ focal cortical dysplasia; $\mathrm{ReHo}=$ regional homogeneity; $\mathrm{WE}=$ wavelet entropy

A focal cortical dysplasia (FCD) is a congenital malformation of cortical development and a frequent cause of intractable epilepsy in children and adults. ${ }^{1}$ A complete resection of the epileptogenic zone is required to become seizure-free; therefore, an accurate visualization by neuroimaging is crucial for surgical planning. Histopathologic examination of surgical specimens has

Received August 31, 2017; accepted after revision April 1, 2018.

From the Department of Radiology and Nuclear Medicine (L.G., P.A.M.H., R.M.H.B., J.F.A.J., W.H.B.) and School for Mental Health and Neuroscience (P.A.M.H., J.F.A.J., W.H.B.), Maastricht University Medical Center, Maastricht, the Netherlands; and Department of Electrical Engineering (R.M.H.B.), Eindhoven University of Technology, Eindhoven, the Netherlands.

This work was partly supported by a grant from the Dutch National Epilepsy Fund $10-08$, and the study is registered in the Dutch Trial Registry (http://www. trialregister.nl/trialreg/admin/rctview.asp?TC $=2315$ ).

Please address correspondence to Walter H. Backes, PhD, Departments of Radiology and Nuclear Medicine, Maastricht University Medical Center (MUMC+), P.O. Box 5800, 6202 AZ Maastricht, the Netherlands; e-mail: w.backes@mumc.nl

http://dx.doi.org/10.3174/ajnr.A5684 demonstrated that $80 \%$ of patients who had a complete resection become seizure-free, compared with only $20 \%$ who had incomplete resections. ${ }^{2}$ The most frequently mentioned cause of unsuccessful surgical treatment is invisibility of lesion boundaries on imaging modalities. ${ }^{3}$

The computer-aided diagnostic approach using morphologic characteristics such as focal cortical thickening or thinning, areas of focal brain atrophy, gray-white junction blurring, and increased signal on T2- and fluid-attenuated inversion recoveryweighted images assists in improving lesion detection. ${ }^{4}$ However, where the exact borders reside and whether the lesion and the perilesional zone are functionally normal or abnormal often remains unknown. ${ }^{5,6}$ Therefore, we proposed exploring a physiologic image contrast in FCD, in addition to the clinically used morphologic contrasts.

Simultaneous electroencephalography (EEG)-fMRI studies revealed that interictal epileptiform discharge-related hemodynamic changes were observed beyond the visible lesion on struc- 


\begin{tabular}{|c|c|c|c|c|}
\hline No. & Age/Sex & $\begin{array}{l}\text { Seizure } \\
\text { Frequency }^{\mathrm{a}}\end{array}$ & FCD Location & MRI Structural Image Findings \\
\hline 1 & $30 / F$ & High & Right insula & $\begin{array}{l}\text { Transmantle sign, gray-white matter blurring, cortical thickening, } \\
\text { T2-FLAIR hyperintensity }\end{array}$ \\
\hline 2 & $29 / F$ & High & Right superior frontal & $\begin{array}{l}\text { Transmantle sign, gray-white matter blurring, cortical thickening, } \\
\text { T2-FLAIR hyperintensity }\end{array}$ \\
\hline 3 & $33 / \mathrm{M}$ & Low & Right inferior frontal & Transmantle sign, gray-white matter blurring, T2-FLAIR hyperintensity \\
\hline 4 & $47 / F$ & High & Right precentral & Transmantle sign, gray-white matter blurring, T2-FLAIR hyperintensity \\
\hline 5 & $21 / \mathrm{M}$ & Low & Left postcentral & Gray-white matter blurring, cortical thickening, T2-FLAIR hyperintensity \\
\hline 6 & $47 / \mathrm{M}$ & High & Left posterior cingulate & $\begin{array}{l}\text { Transmantle sign, gray-white matter blurring, cortical thickening, } \\
\text { T2-FLAIR hyperintensity }\end{array}$ \\
\hline 7 & $43 / \mathrm{M}$ & High & Left caudal middle frontal & $\begin{array}{l}\text { Transmantle sign, gray-white matter blurring, cortical thickening, } \\
\text { T2-FLAIR hyperintensity }\end{array}$ \\
\hline 8 & $26 / M$ & Low & Left rostral middle frontal & $\begin{array}{l}\text { Transmantle sign, gray-white matter blurring, cortical thickening, } \\
\text { T2-FLAIR hyperintensity }\end{array}$ \\
\hline 9 & $21 / F$ & Low & Caudal anterior cingulate & $\begin{array}{l}\text { Transmantle sign, gray-white matter blurring, cortical thickening, } \\
\text { T2-FLAIR hyperintensity }\end{array}$ \\
\hline 10 & $21 / M$ & High & Right supramarginal & $\begin{array}{l}\text { Transmantle sign, gray-white matter blurring, cortical thickening, } \\
\text { T2-FLAIR hyperintensity }\end{array}$ \\
\hline 11 & $21 / \mathrm{M}$ & Low & Right insular & $\begin{array}{l}\text { Transmantle sign, gray-white matter blurring, cortical thickening, } \\
\text { T2-FLAIR hyperintensity }\end{array}$ \\
\hline 12 & $27 / M$ & Low & Left precentral & $\begin{array}{l}\text { Transmantle sign, gray-white matter blurring, cortical thickening, } \\
\text { T2-FLAIR hyperintensity }\end{array}$ \\
\hline 13 & $54 / \mathrm{M}$ & High & Right rostral middle frontal & $\begin{array}{l}\text { Transmantle sign, gray-white matter blurring, cortical thickening, } \\
\text { T2-FLAIR hyperintensity }\end{array}$ \\
\hline 14 & $25 / M$ & None & Left inferior parietal & Gray-white matter blurring, cortical thickening, T2-FLAIR hyperintensity \\
\hline
\end{tabular}

a Seizure frequency: low, 1 per month; high, $>1$ per week.

tural MR imaging, and surgical outcomes were better using EEGfMRI in addition to structural MR imaging and intracranial EEG. ${ }^{7}$ Electrophysiologic examinations have revealed epileptogenicity, not only in the lesion but also in perilesional areas. ${ }^{8}$ To our knowledge, no systematic imaging study has explored the physiology of the immediate vicinity of FCDs, which may be relevant for resection planning and outcome prediction.

Using resting-state functional MR imaging, we assessed the time signature of spontaneous blood oxygen level-dependent (BOLD) fluctuations in patients with FCD. We evaluated 3 known BOLD measures to characterize the spontaneous fluctuations: 1) the amplitude (fractional amplitude of low frequency fluctuations [fALFF]) as a measure of oscillation strength, 2) regional homogeneity ( $\mathrm{ReHo}$ ) as a measure of local signal similarity, and 3) wavelet entropy (WE) as a measure of disorder/order in BOLD time-series.

The same functional MR imaging dataset was previously used to show abnormalities in the spatial profile of functional connectivity of FCDs beyond the MR imaging-visible lesion. ${ }^{9}$ That study used a seed-based functional connectivity approach to determine the interregional correlations with remote brain regions, whereas in the current study, we characterized the intrinsic BOLD fluctuations in FCD lesions with local spatiotemporal measures (fALFF, ReHo, and WE). The objective of this study was to determine whether functional abnormalities arise in spontaneous BOLD fluctuations of FCD lesions and proximal regions in comparison with normal-appearing brain tissue.

\section{MATERIALS AND METHODS Subjects}

The study was approved by the ethics committees of the 2 participating medical institutions. Informed written consent was obtained from all subjects. Fourteen patients with FCD-related epi- lepsy were recruited (mean age, $32 \pm 11$ years; 10 men), as well as 16 age- and sex-matched healthy controls (mean age, $35 \pm 9$ years; 7 men). Structural MR images were rated by neuroradiologists with $>20$ years of experience. The clinical diagnosis was based on concordance among seizure semiology, EEG findings, and neuroimaging. ${ }^{1}$ Briefly, this involved recurrent stereotyped seizures and focal interictal and/or ictal EEG abnormalities that coincided with an FCD-concordant lesion on MR imaging. ${ }^{10}$ Relevant imaging features included, among others, an abnormal gyral pattern, increased cortical thickness, the transmantle sign, and blurring of the gray-white matter interface. ${ }^{11}$ None of the patients included in this study had dual pathology. Table 1 lists the lesion characteristics per patient. For 5 patients, surgery and histopathology information were also available, which further confirmed the diagnosis of FCD.

\section{MR Imaging}

All subjects underwent structural and functional MR imaging at 3T (Achieva; Philips Healthcare, Best, the Netherlands) using an 8 -element receive-only head coil. Structural imaging involved a T1-weighted scan: 3D fast spoiled gradient-echo sequence; TE/ TR/TI, 3.8/8.3/1022 milliseconds; voxel size, $1 \times 1 \times 1 \mathrm{~mm}^{3}$; duration, 7.5 minutes. In addition, a FLAIR sequence was used (3D turbo spin-echo; TE/TR/TI, 330/8000/2400 milliseconds; $0.4 \times 0.4 \mathrm{~mm}^{2}$ pixel size; 0.6 - $\mathrm{mm}$-thick axial slices; duration, 8 minutes). Functional MR imaging involved a BOLD T2*weighted task-free scan, for which the participants were instructed to close their eyes, lie still, and think of nothing in particular. The settings were the following: single-shot echoplanar imaging sequence; TE/TR, 35/2000 milliseconds; $2 \times 2$ $\mathrm{mm}^{2}$ pixel size; $4-\mathrm{mm}$ thick axial slices; 195 dynamics; duration, 6.5 minutes. 


\section{Image Processing}

Functional images were corrected for slice-timing differences and head displacements, coregistered to the anatomic template, and smoothed with an 8-mm kernel (full width at half maximum) using computational software (Statistical Parametric Mapping, SPM8; http://www.fil.ion.ucl.ac.uk/spm/software/spm12). Any signal drifts across time were corrected by removing the very-lowfrequency components (<10 MHz) (FSL 3.0; http://www.fmrib. ox.ac.uk/fsl). To correct for physiologic fluctuations, we included the time-series from the CSF and white matter as covariates in the linear regression analysis. ${ }^{12}$ Gray matter, white matter, and CSF voxels were segmented from the T1-weighted images (FreeSurfer software, Version 5.3.0; http://surfer.nmr. mgh.harvard.edu).

\section{Fractional Amplitude of Low-Frequency Fluctuations}

The signal time-series of each voxel was standardized and Fourier-transformed to the frequency domain, and the power spectrum was calculated in specific frequency sub-bands. The fALFF is computed per voxel as the ratio of the power spectrum in a specific sub-band $(10-80 \mathrm{MHz})$ to the full range $(0-250 \mathrm{MHz})^{13}$ (Resting-State fMRI Data Analysis Toolkit [REST], Version 1.8; http:// www.rfmri.org/REST). This ratio helps to eliminate the confounding influence of the relatively strong signal of the pulsating CSF and physiologic noise. ${ }^{13}$ The fALFF was computed in 4 predefined frequency sub-bands: $10-27 \mathrm{MHz}$ (slow-5), 27-73 MHz (slow-4), 73-198 MHz (slow-3), and 198-250 MHz (slow-2). ${ }^{14}$

\section{Regional Homogeneity}

The Kendall coefficient of concordance was used to calculate the ReHo of the time-series of a voxel with its 26 (immediate) neighbor voxels (REST Toolkit, Version 1.8) as proposed by Zang et al. ${ }^{15}$

ReHo measures the similarity in contiguous voxels of the time-series and was averaged over all gray matter voxels per ROI. While most functional imaging analysis methods rely on a priori knowledge of a signal model, ReHo is a voxel-by-voxel, datadriven approach that requires no prior knowledge of the experimental design.

\section{Wavelet Entropy}

The wavelet entropy is computed using the discrete wavelet transform of the time-series. ${ }^{16}$ Unlike the Fourier transform or shorttime Fourier transform, the nonstationary components in the BOLD time-series are detected by the discrete wavelet transform (Daubechies- 4 wavelet) by means of the convolution with the time-shifted and scaled mother wavelet function. The resulting sub-bands are squared and integrated across time to obtain the energy $E_{\mathrm{i}}$ per sub-band i, from which the WE over the sub-bands is calculated as $\mathrm{WE}=-\Sigma p_{\mathrm{i}} \ln p_{\mathrm{i}}$, with $p_{\mathrm{i}}=E_{\mathrm{i}} / \Sigma E_{\mathrm{i}}$, the relative energy per sub-band. WE is a measure of lack of structure, thus randomness, over the wavelet sub-band. A typical BOLD signal represents a frequency structure in which the energy roughly decreases as a function of frequency (sub-band) due to the low-pass nature of the blood signal. When the distribution of energy over frequency sub-bands becomes more equal (ie, more random), WE increases. ${ }^{17}$

\section{Image Analysis}

FCDs were manually marked on the structural images by an experienced neuroradiologist on FLAIR images. These ROIs were spatially transformed to the functional images. Three proximal regions of FCD were selected at a 1-, 2-, and 3-cm distance from the lesion border, as 1-cm-thick shells. To reduce physiologic variation, we used the entire normal-appearing cortex of the contralateral hemisphere for normalizing all BOLD measures. In healthy controls, the BOLD measures were obtained from the anatomic atlas regions that spatially corresponded to the lesions in patients (using FreeSurfer software).

\section{Statistical Analysis}

Region-averaged BOLD measures of the FCD lesions and the homotopic contralateral cortex were reported as absolute (ie, not normalized to the contralateral cortex) values. In addition, normalized values were given as a ratio between the region-averaged BOLD value (fALFF, ReHo, or WE) and the mean measure of all voxels in the entire normal-appearing cortex of the contralateral hemisphere.

The Student 2-sample $t$ test was used to reveal any differences between the patients with FCD and control subjects. The paired $t$ test was used for statistical assessment of the FCD region, proximal regions, and contralateral homotopic cortex. Statistical significance was inferred at $P<.05$.

A threshold was derived from the results of the healthy control group (reference value). This threshold served to determine which and how many patients had abnormal BOLD values (ie, beyond the $95 \%$ confidence intervals of the healthy control group) in the lesion and proximal regions.

\section{RESULTS}

Figure 1 shows an FCD lesion on a FLAIR image with cortical maps of fALFF, ReHo, and WE of the FCD lesion and proximal cortex. In this example, decreased fALFF, increased ReHo, and increased WE were observed in the FCD lesion and the proximal regions.

\section{Absolute BOLD Measures}

Table 2 lists the absolute BOLD measures. The fALFF of the FCD lesions was significantly lower and wavelet entropy was significantly higher than the contralateral homotopic cortex and the same region in the control subjects. ReHo showed no significant differences.

\section{Normalized Fractional Amplitude of Low-Frequency Fluctuations}

Figure 2 provides the fALFF as a function of frequency in the range of $10-250 \mathrm{MHz}$ for the FCD lesion, proximal regions, and controls. Only for the slow-5 sub-band (10-27 MHz) was the amplitude of the FCD lesions significantly lower than for the control subjects $(P<.001)$. For this sub-band, all 3 proximal regions showed lower amplitudes than in controls. The most distant shell at $3 \mathrm{~cm}$ showed a significantly higher fALFF than the more proximal regions ( 1 and $2 \mathrm{~cm}$ ).

Patients showed a significantly lower normalized fALFF in the FCD lesion and the closer proximal regions (at 1 and $2 \mathrm{~cm}$ ) rela- 


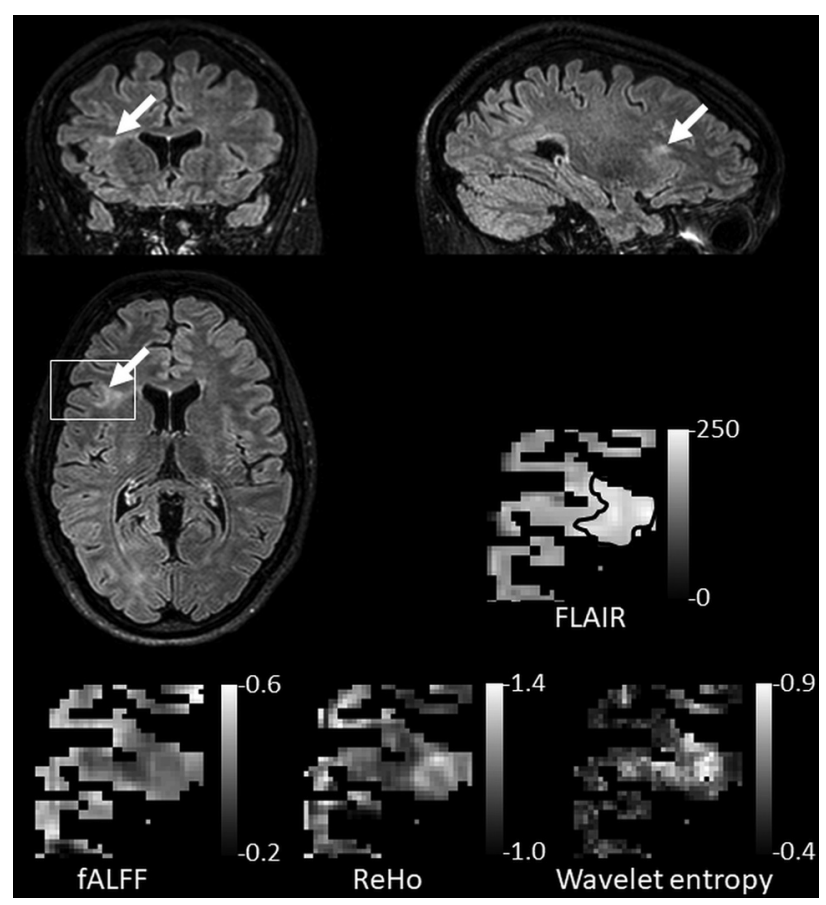

FIG 1. Sample orthogonal FLAIR images depicting an FCD lesion (arrows) of a 30-year-old woman. Inserts show the magnification of the lesion on FLAIR contrast and cortical parameter maps of fALFF, regional homogeneity, and wavelet entropy. Increased regional homogeneity, wavelet entropy, and decreased fALFF were observed in the FCD lesion and the immediate perilesional regions in comparison with the regions more distinct from the FCD. Only gray matter is visualized; white matter is masked out.

Table 2: Absolute BOLD measures in the FCD lesion, the contralateral homotopic cortex, and healthy controls ${ }^{a}$

\begin{tabular}{lccc}
\hline $\begin{array}{l}\text { Absolute } \\
\text { Measure }\end{array}$ & Lesion & $\begin{array}{c}\text { Contralateral } \\
\text { Homotopic } \\
\text { Cortex }\end{array}$ & Controls \\
\hline fALFF & $0.327 \pm 0.007^{\mathrm{b}}$ & $0.352 \pm 0.008$ & $0.358 \pm 0.008$ \\
ReHo & $0.348 \pm 0.032$ & $0.292 \pm 0.031$ & $0.310 \pm 0.024$ \\
WE & $1.245 \pm 0.005^{\mathrm{b}}$ & $1.227 \pm 0.004$ & $1.227 \pm 0.003$ \\
\hline
\end{tabular}

${ }^{a}$ Data are mean \pm standard error.

b Significant differences $(P<.05)$ with the contralateral homotopic cortex and the same region in controls, respectively.

tive to the contralateral homotopic cortex (FCD lesion: $P<.001$; proximal at 1 and $2 \mathrm{~cm}: P<.02)$ and the same regions in the control subjects (FCD lesion: $P<.001$; proximal at 1 and $2 \mathrm{~cm}$ : $P<.01$ ) (Table 3). Thirteen of 14 patients showed abnormal (lower) fALFF in the lesions, and 11 of these 13 patients showed abnormal fALFF up to $2 \mathrm{~cm}$ from the boundary (Table 4). Six patients showed abnormal fALFF in the contralateral homotopic cortex.

\section{Normalized Regional Homogeneity}

The normalized ReHo was significantly higher in FCD lesions compared with the contralateral homotopic cortex in patients $(P<.01)$ and control subjects $(P<.01)$. None of the proximal regions showed significant differences. The mean ReHo increased with the distance in the proximal regions (Table 3). Eleven of 14 patients showed abnormal (higher) ReHo in the lesion, and 5 of these patients showed abnormal ReHo up to $2 \mathrm{~cm}$ from the boundary (Table 4). Four patients showed abnormal fALFF in the contralateral homotopic cortex.

\section{Normalized Wavelet Entropy}

The normalized WE for the FCD lesion and the closer proximal regions (at 1 or $2 \mathrm{~cm}$ ) was significantly higher than the contralateral homotopic cortex (FCD lesion: $P<.01$; proximal at 1 and 2 $\mathrm{cm}: P<.05)$ and the same region in healthy controls (FCD lesion: $P<.01$; proximal at 1 and $2 \mathrm{~cm}: P<.05$ ) (Table 3 ). Twelve of 14 patients showed abnormal (higher) WE in the lesion, and 10 of these patients showed abnormal WE up to $2 \mathrm{~cm}$ from the boundary (Table 4). Five patients showed abnormal WE in the contralateral homotopic cortex.

\section{DISCUSSION}

In this study, we set out to determine physiologic abnormalities of the BOLD signal time-series in FCD lesions of patients with epilepsy. The most suitable BOLD measures were obtained by normalizing lesion or perilesional values to the contralateral homotopic cortex. Patients with FCD showed significantly decreased oscillation amplitudes (normalized fALFF), decreased spatiotemporal heterogeneity (ie, increased normalized ReHo), and increased temporal heterogeneity (ie, increased normalized WE) in the FCD lesions compared with healthy controls. The most striking result was that abnormal BOLD fluctuations were also manifest in regions adjacent to the structurally visible borders of the lesions.

\section{BOLD Abnormalities in FCD Lesions}

Decreased BOLD amplitude (normalized fALFF) and increased temporal heterogeneity (increased normalized WE) suggest reduced neuronal activity or neurovascular coupling in the lesions. Suppression of activity in the FCD lesion was also previously reported in the intracranial EEG, where interrupted pseudoperiodic spikes were reported. ${ }^{18}$

There was also significantly increased normalized ReHo observed in patients with FCD lesions relative to healthy controls. This could be due to the lower amplitude, which also attenuates differences and randomness in BOLD signals within FCD lesions. The increase in normalized ReHo increases the similarity of the signals and thus increases ReHo.

\section{Perilesional Functional Abnormalities}

All 3 BOLD measures and the wavelet spectrum showed that the abnormalities extend beyond the visible lesion border on FLAIR images. An increase in normalized WE in the FCD lesion and the $2-\mathrm{cm}$ perilesional zone was observed, indicating a more equalized frequency distribution over the sub-bands for the FCD lesion and the perilesional zone, which implies a less pronounced frequency structure. The normalized fALFF measure also showed reduction of activity, indicating that irregularities of the BOLD fluctuations in the perilesional zone may be related to some form of epilepsyrelated activity of the impaired brain. ${ }^{7,19}$ However, from the current observations in patients with epilepsy, who were well-treated by medication and/or surgery, we cannot infer whether the perilesional activity acts as an epileptogenic or inhibitory mechanism.

Also previous electrophysiologic investigations showed that interictal epileptic discharges extend beyond the FCD visible le- 
sion (ie, in perilesional regions). ${ }^{20,21}$ Urrestarazu et al ${ }^{22}$ reported that a very high rate of fast ripples in the EEG signal was observed in the perilesional area, which was adjacent to the seizure-onset area. The fast ripples in the perilesional zone might be an indicator of potential epileptogenicity, which Cohen-Gadol et $\mathrm{al}^{23}$ suggested could possibly turn into a seizure focus after the removal of the primary seizure-onset zone. Other studies further suggested that removal of the entire lesion, including surrounding interictally active tissue, would be necessary to achieve long-term seizure relief. ${ }^{24,25}$

The results indicated variability among patients because not all patients showed BOLD abnormalities up to $2 \mathrm{~cm}$ from the lesion boundaries and only a few patients showed abnormalities beyond $2 \mathrm{~cm}$. This intersubject variation suggests that different pathologic mechanisms might be at work; for instance, excitotoxic effects could lead to variations in the BOLD signal. The variable findings may also reflect the heterogeneity of a clinical sample of patients with FCD, with variations in the frequency of epileptiform discharges and antiepileptic drug use. The results presented in this study are in line with the findings presented in a functional connectivity study, in which $11 / 14$ patients (a similar number of patients showed abnormality using normalized fALFF and normalized WE) showed abnormality in FCD perilesional regions ${ }^{9}$ on the same dataset.

Note that because structural findings like the transmantle sign and gray-white matter blurring potentially infer an indeterminate lesion border, which may influence the exact values of the BOLD measures we obtained in individuals. In this study, we delineated

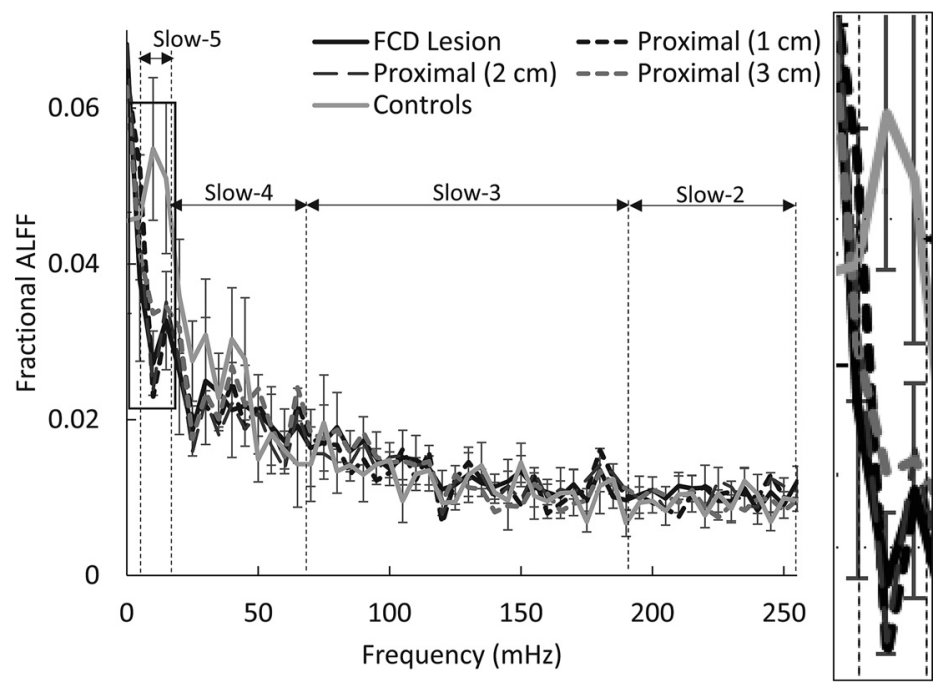

FIG 2. Fractional amplitude of low frequency fluctuations as a function of frequency in the range of $10-250 \mathrm{MHz}$. Only the lowest sub-band (ie, slow-5; $10-27 \mathrm{MHz}$, magnified) showed significantly lower amplitudes in the FCD lesion relative to the controls. Error bars represent the standard error of mean. the lesion border on the hyperintensities of the FLAIR images, which were present for all subjects. It is unlikely that individual uncertainties in border definition would strongly influence our results because we present group average results.

\section{Clinical Perspective}

The most interesting finding of this study is that structural boundaries are not the same as functional boundaries obtained by the physiologic BOLD contrast for the FCD lesions. Reports indicate that on average, $62 \%$ of patients with cortical dysplasia are seizure-free after an operation. ${ }^{2,26}$ Our findings show that functional abnormalities, derived from spontaneous BOLD fluctuations, were seen up to $2 \mathrm{~cm}$ distant from the radiologically visible boundary of the FCD lesion. These findings could be critical information for surgery planning of FCD lesions.

Recent findings show that removing as much as possible of the aberrant cortex improves seizure-free outcome. ${ }^{2}$ The presented BOLD method can possibly be used to help noninvasively assess the functional extent of FCD lesions and has the potential to unveil lesion areas that could be missed by structural MR imaging in a surgical work-up.

\section{Study Considerations}

Because the 2 brain hemispheres are intrinsically connected, the BOLD signal of the contralateral homotopic cortex is also likely to be affected by the abnormality caused by FCD. This abnormality was noticed because some patients showed increased normalized WE and decreased normalized fALFF in comparison with controls in the contralateral homotopic cortex.

Functional imaging has been linked to electrophysiology, in a previous study in which epileptiform spikes were mapped to the brain using simultaneously acquired EEG-fMRI. ${ }^{7}$ The current study results need to be verified by comparing BOLD measures on functional MR imaging data from patients who become seizure-free after an operation with measures of those who have recurrent seizures. Ideally, this comparison should be supplemented with histopathology according to the most recent classification, ${ }^{1,27}$ which we could not fully provide. Similarly, our findings on the abnormal BOLD fluctuations in the perilesional zone need to be evaluated against intracranial EGG recordings in suitable patient groups.

Table 3: The normalized BOLD measures in the FCD lesion, perilesional regions, contralateral homotopic region, and healthy controls ${ }^{\mathrm{a}}$

\begin{tabular}{lccccrr}
\hline \multirow{2}{*}{$\begin{array}{c}\text { Normalized } \\
\text { Measure }\end{array}$} & Lesion & $\mathbf{1} \mathbf{c m}$ & $\mathbf{2} \mathbf{c m}$ & \multicolumn{2}{c}{ Perilesional Zones } & \multicolumn{2}{c}{$\begin{array}{c}\text { Contralateral } \\
\text { Homotopic }\end{array}$} & $\begin{array}{c}\text { Control } \\
\text { Subjects }\end{array}$ \\
\cline { 2 - 5 } fALFF & $0.922 \pm 0.016^{\mathrm{b}}$ & $0.941 \pm 0.014^{\mathrm{b}}$ & $0.947 \pm 0.014^{\mathrm{b}}$ & $0.981 \pm 0.013$ & $0.983 \pm 0.012$ & $1.005 \pm 0.009$ \\
ReHo & $1.206 \pm 0.066^{\mathrm{b}}$ & $0.992 \pm 0.032$ & $0.991 \pm 0.036$ & $1.021 \pm 0.034$ & $1.023 \pm 0.032$ & $1.020 \pm 0.022$ \\
WE & $1.018 \pm 0.004^{\mathrm{b}}$ & $1.013 \pm 0.003^{\mathrm{b}}$ & $1.009 \pm 0.003^{\mathrm{b}}$ & $1.003 \pm 0.003$ & $1.003 \pm 0.002$ & $1.002 \pm 0.002$ \\
\hline
\end{tabular}

${ }^{a}$ Data are mean \pm standard error. Normalized fALFF was significantly decreased, while WE was significantly higher in FCD lesions and perilesional zones (up to $2 \mathrm{~cm}$ ) than in the contralateral homotopic cortex and control subjects. ReHo was significantly higher in FCD lesions. BOLD measures were normalized with the entire contralateral cortex.

${ }^{b}$ Significant differences $(P<.05)$ with the contralateral homotopic cortex and the same region in controls, respectively. 
Table 4: Number of patients with measures outside the $95 \%$ confidence interval of the normative (healthy control) values ${ }^{a}$

\begin{tabular}{|c|c|c|c|c|c|}
\hline \multirow{2}{*}{$\begin{array}{c}\text { Normalized } \\
\text { Measure }\end{array}$} & \multirow[b]{2}{*}{ Lesion } & \multicolumn{3}{|c|}{ Perilesional Zone } & \multirow{2}{*}{$\begin{array}{c}\text { Contralateral } \\
\text { Homotopic } \\
\text { Cortex }\end{array}$} \\
\hline & & $1 \mathrm{~cm}$ & $2 \mathrm{~cm}$ & $3 \mathrm{~cm}$ & \\
\hline fALFF & $13(93)$ & $12(86)$ & $11(79)$ & $6(43)$ & $6(43)$ \\
\hline ReHo & $11(79)$ & $5(36)$ & $5(36)$ & $4(29)$ & $4(29)$ \\
\hline WE & $12(86)$ & $11(79)$ & 10 (71) & $4(29)$ & $5(36)$ \\
\hline
\end{tabular}

${ }^{a}$ Data are the number of patients (percentage)

\section{CONCLUSIONS}

In this study, it was shown that functional boundaries of FCD extend beyond boundaries visible on structural MR imaging. Our findings show that functional abnormalities, in terms of attenuated and more disordered BOLD fluctuations, were seen up to 2 $\mathrm{cm}$ away from the radiologically visible boundary of the lesion. This information could be critical for surgery planning of FCD lesions, which needs to be validated with intracranial EEG recordings, because BOLD findings in the lesional and perilesional areas could reflect metabolic/hemodynamic changes due to epileptic discharges.

Disclosures: Paul A.M. Hofman—RELATED: Grant: Dutch Epilepsy Fund, Comments: Project $10-8 .{ }^{*}$ *Money paid to the institution.

\section{REFERENCES}

1. Blümcke I, Thom M, Aronica E, et al. The clinicopathological spectrum of focal cortical dysplasias: a consensus classification proposed by an ad hoc Task Force of the ILAE Diagnostic Methods Commission. Epilepsia 2011;52:158-74 CrossRef Medline

2. Hauptman JS, Mathern GW. Surgical treatment of epilepsy associated with cortical dysplasia: 2012 update. Epilepsia 2012;53(Suppl 4):98-104 CrossRef Medline

3. Winston GP, Micallef C, Kendell BE, et al. The value of repeat neuroimaging for epilepsy at a tertiary referral centre: 16 years of experience. Epilepsy Res 2013;105:349-55 CrossRef Medline

4. Yagishita A, Arai N, Maehara T, et al. Focal cortical dysplasia: appearance on MR images. Radiology 1997;203:553-59 CrossRef Medline

5. Jiang YJ, Ang LC, Blume WT. Extent of EEG epileptiform pattern distribution in "focal" cortical dysplasia. J Clin Neurophysiol 2010; 27:309-11 CrossRef Medline

6. Tassi L, Colombo N, Garbelli R, et al. Focal cortical dysplasia: neuropathological subtypes, EEG, neuroimaging and surgical outcome. Brain 2002;125(Pt 8):1719-32 Medline

7. Thornton R, Vulliemoz S, Rodionov R, et al. Epileptic networks in focal cortical dysplasia revealed using electroencephalography-functional magnetic resonance imaging. Ann Neurol 2011;70:822-37 CrossRef Medline

8. Hodozuka A, Tsuda H, Hashizume K, et al. Focal cortical dysplasia: pathophysiological approach. Childs Nerv Syst 2006;22:827-33 CrossRef Medline

9. Besseling RMH, Jansen JFA, de Louw AJA, et al. Abnormal profiles of local functional connectivity proximal to focal cortical dysplasias. PLoS One 2016;11:e0166022 CrossRef Medline

10. Chassoux F, Landré E, Mellerio C, et al. Type II focal cortical dysplasia: electroclinical phenotype and surgical outcome related to imaging. Epilepsia 2012;53:349-58 CrossRef Medline

11. Hofman PAM, Fitt GJ, Harvey AS, et al. Bottom-of-sulcus dysplasia: imaging features. AJR Am J Roentgenol 2011;196:881-85 CrossRef Medline

12. Zou QH, Zhu CZ, Yang Y, et al. An improved approach to detection of amplitude of low-frequency fluctuation (ALFF) for resting-state fMRI: fractional ALFF. J Neurosci Methods 2008;172:137-41 CrossRef Medline

13. Zang YF, He Y, Zhu CZ,et al. Altered baseline brain activity in children with ADHD revealed by resting-state functional MRI. Brain Dev 2007;29:83-91 CrossRef Medline

14. Wang Z, Zhang Z, Liao W, et al. Frequency-dependent amplitude alterations of resting-state spontaneous fluctuations in idiopathic generalized epilepsy. Epilepsy Res 2014;108:853-60 CrossRef Medline

15. Zang $\mathrm{Y}$, Jiang $\mathrm{T}, \mathrm{Lu} \mathrm{Y}$, et al. Regional homogeneity approach to fMRI data analysis. Neuroimage 2004;22:394-400 CrossRef Medline

16. Rosso OA. Entropy changes in brain function. Int J Psychophysiol 2007;64:75-80 CrossRef Medline

17. Gupta L, Jansen JFA, Hofman PAM, et al. Wavelet entropy of BOLD time series: an application to Rolandic epilepsy. J Magn Reson Imaging 2017;46:1728-37 CrossRef Medline

18. Menezes Cordeiro I, von Ellenrieder N, Zazubovits N, et al. Sleep influences the intracerebral EEG pattern of focal cortical dysplasia. Epilepsy Res 2015;113:132-39 CrossRef Medline

19. Gotman J. Epileptic networks studied with EEG-fMRI. Epilepsia 2008;49(Suppl 3):42-51 CrossRef Medline

20. Tassi L, Colombo N, Cossu M, et al. Electroclinical, MRI and neuropathological study of 10 patients with nodular heterotopia, with surgical outcomes. Brain 2005;128:321-37 Medline

21. Palmini A, Gambardella A, Andermann F, et al. Intrinsic epileptogenicity of human dysplastic cortex as suggested by corticography and surgical results. Ann Neurol 1995;37:476-87 CrossRef Medline

22. Urrestarazu E, Chander R, Dubeau F, et al. Interictal high-frequency oscillations $(100-500 \mathrm{~Hz})$ in the intracerebral EEG of epileptic patients. Brain 2007;130:2354-66 CrossRef Medline

23. Cohen-Gadol AA, Ozduman K, Bronen RA, et al. Long-term outcome after epilepsy surgery for focal cortical dysplasia. J Neurosurg 2004;101:55-65 CrossRef Medline

24. Hader WJ, Mackay M, Otsubo H, et al. Cortical dysplastic lesions in children with intractable epilepsy: role of complete resection. J Neurosurg 2004;100(2 Suppl Pediatrics):110-17 CrossRef Medline

25. Alexandre V Jr, Walz R, Bianchin MM, et al. Seizure outcome after surgery for epilepsy due to focal cortical dysplastic lesions. Seizure 2006; 15:420-27 CrossRef Medline

26. Phi JH, Cho BK, Wang -C, et al. Longitudinal analyses of the surgical outcomes of pediatric epilepsy patients with focal cortical dysplasia. J Neurosurg Pediatr 2010;6:49-56 CrossRef Medline

27. Najm IM, Sarnat HB, Blümcke I. Review: the International Consensus Classification of Focal Cortical Dysplasia-a critical update 2018. Neuropathol Appl Neurobiol 2018;44:18-31 CrossRef Medline 